\title{
De nutrientes a patrones alimentarios: cambio de paradigma en el abordaje
}

nutricional de las enfermedades cardiovasculares

DOI: 10.17533/udea.penh.v22n1a08

PERSPECTIVAS EN NUTRICIÓN HUMANA

ISSN 0124-4108

Escuela de Nutrición y Dietética, Universidad de Antioquia. Medellín, Colombia

Vol. 22, N. ${ }^{\circ}$, enero-junio de 2020, pp. 101-111.

Artículo recibido: 13 de noviembre de 2019

Aprobado: 24 de septiembre de 2020

\section{Lorena Viola ${ }^{1 *}$; Georgina Noel Marchiori²; María Daniela Defagó ${ }^{3}$}

\section{- - - Resumen}

Antecedentes: en la actualidad, existen controversias sobre las recomendaciones nutricionales en las enfermedades cardiovasculares. Objetivo: se propuso analizar, en una línea temporal, la transición histórica y los cambios de paradigmas en el estudio de la alimentación y de la enfermedad cardiovascular a fin de avanzar en el campo de la epidemiología nutricional, contemplando el patrón alimentario y del estilo de vida. Reflexión: históricamente, los estudios que investigaron la asociación entre alimentación y riesgo cardiovascular se centraron en calorías o nutrientes como factores aislados; sin embargo, esto ha sido problemático para el abordaje del fenómeno multidimensional de las enfermedades crónicas. En los últimos años, se ha propuesto el análisis de los patrones alimentarios y el grado de procesamiento de alimentos, que incluye la combinación global de alimentos y el efecto sinérgico de ciertos nutrientes. Conclusión: la epidemiología nutricional evidenció que el enfoque en calorías y nutrientes no es suficiente en el análisis de las enfermedades crónicas, y que las enfermedades cardiovasculares - fenómeno multicausal y complejo— están influenciadas por los efectos sinérgicos de alimentos y nutrientes, la calidad de los alimentos consumidos, el grado de procesamiento industrial, los entornos alimentarios y los patrones dietarios en general.

Palabras clave: enfermedad cardiovascular, nutrientes, alimentos, ultraprocesados, dieta, patrones alimentarios

1 Autor de correspondencia. Licenciada en Nutrición. Doctorando en Ciencias de la Salud, Facultad de Ciencias Médicas, Universidad Nacional de Córdoba, Argentina. Edificio Escuelas, 2. Piso, Av. Enrique Barros s/n, CP 5016, Córdoba, Argentina. Teléfono: (0351) 535-3687. Orcid: https://orcid.org/0000-0001-9539-2761. Correo electrónico: violalorena@ gmail.com

2 Licenciada en Nutrición. Escuela de Nutrición. Doctorando en Ciencias de la Salud Facultad de Ciencias Médicas, Universidad Nacional de Córdoba, Argentina. Instituto de Investigaciones en Ciencias de la Salud (INICSA-CONICET). Córdoba, Argentina. Correo electrónico: georginamarchiori@gmial.com

3 Licenciada en Nutrición. Magíster en Efectividad Clínica. Doctora en Ciencias de la Salud. Escuela de Nutrición, Facultad de Ciencias Médicas, Universidad Nacional de Córdoba, Argentina. Instituto de Investigaciones en Ciencias de la Salud (INICSA-CONICET). Córdoba, Argentina. Orcid: https://orcid.org/0000-0002-8878-3067. Correo electrónico: mddefago@gmail.com

Cómo citar este artículo: Viola L; Noel Marchiori GN; Daniela Defagó MD. De nutrientes a patrones alimentarios: cambio de paradigma en el abordaje nutricional de las enfermedades cardiovasculares. Perspect Nutr Humana. 2020;22:101-111. DOI: 10.17533/udea.penh.v22n1a08 
Nutrientes, patrones alimentarios y enfermedad cardiovascular

\section{From Nutrients to Eating Patterns:A Paradigm Shift in the Nutritional Approach to Cardiovascular Diseases}

\section{Abstract}

Background: At present, controversies exist around nutrition recommendations for cardiovascular diseases. Objective: We proposed to analyze over time the historical transition and paradigm shifts in the study of diet and cardiovascular disease in order to advance the field of nutritional epidemiology, considering dietary patterns and lifestyles. Reflection: Historically, studies that investigate the association between diet and cardiovascular risk have centered on calories or nutrients as isolated factors. However, this has been problematic in addressing the multidimensional phenomenon of chronic diseases. In the last few years, the analysis of dietary patterns has been proposed along with the degree of food processing, which includes the global combination of foods and the synergistic effects of certain nutrients. Conclusion: Nutritional epidemiology has evidenced that the focus on calories and nutrients is not sufficient for the analysis of chronic diseases. It's also been shown that cardiovascular diseases - both multicausal and complex - are influenced by: synergistic effects of food and nutrients; the quality of foods consumed; the degree of industrial processing; food environments; and dietary patterns in general.

Keywords: Cardiovascular Diseases, Nutrients, Foods, Ultra-Processed, Diet, Food Patterns.

\section{INTRODUCCIÓN}

Las enfermedades cardiovasculares (ECV) son consideradas la primera causa de morbimortalidad a nivel mundial en la actualidad. Se reconoce el importante rol de la alimentación tanto en la prevención como en el tratamiento de estas enfermedades y se considera a la alimentación inadecuada un factor de riesgo modificable en la salud cardiovascular (1). Sin embargo, aún existen ciertas controversias sobre las recomendaciones nutricionales en el manejo de las ECV, los efectos de los diferentes tipos de nutrientes, como ácidos grasos y colesterol, o el análisis de calorías o nutrientes como factores aislados en la salud cardiovascular.

Históricamente, los estudios que investigaron la asociación entre alimentación y riesgo cardiovascular se centraron en los productos alimenticios individuales 0 en nutrientes específicos de la alimentación. En la actualidad, el enfoque en patrones alimentarios permite reflejar la naturaleza multidimensional de las dietas consumidas por la población, examinar la combinación global de los alimentos y los efectos sinérgicos de alimentos y nutrientes (2). Más recientemente, el análisis de alimentos según el grado de procesamiento por el sistema NOVA proporciona una nueva perspectiva del riesgo potencial del consumo de alimentos ultraprocesados (AUP) en la salud cardiovascular (3). El objetivo de la presente reflexión fue analizar, en una línea temporal, los diferentes enfoques en el abordaje nutricional en ECV, el cambio de paradigma en las últimas décadas y la tendencia futura.

\section{REFLEXIÓN}

\section{Enfermedad cardiovascular y cambio de para- digma en nutrición}

La nutrición inadecuada es un factor de riesgo modificable que claramente influye en la morbilidad y la mortalidad por ECV (4). A principios de 1900, el paradigma de deficiencia predominó en el análisis 
de la alimentación, debido a que la escasez de alimentos y la insuficiencia de calorías y nutrientes eran situaciones frecuentes. La modernización de la agricultura, el procesamiento y las formulaciones de alimentos alrededor de la década de 1940 mitigaron las deficiencias de nutrientes en Estados Unidos y en otras naciones de altos ingresos; además, favorecieron el surgimiento de una creciente epidemia de enfermedades crónicas, entre ellas las ECV (5). Después de décadas de énfasis en las enfermedades por deficiencia, el paradigma de un solo nutriente continuó dominando los enfoques de investigación en nutrición, identificando el nutriente relevante y estableciendo recomendaciones nutricionales al respecto (2).

El primer estudio sobre nutrición y ECV data de 1908. En un trabajo experimental, Alexander Ingatowski observó que los conejos alimentados con una dieta rica en colesterol, con base en leche, huevo y carne, desarrollaron aterosclerosis (6). A partir de allí, se iniciaron diversas investigaciones en ECV enfocadas, principalmente, en el papel de las grasas saturadas y el colesterol sobre el riesgo cardiovascular. El estudio del corazón de Framingham, "The Framingham Heart Study" (7), fue el primero a largo plazo que se centró en los factores de riesgo, con una cohorte original de 5209 reclutados entre 1948 y 1952, mientras que el estudio de los siete países de Ancel Keys, "The Seven Countries Study" (8), que comenzó en 1956 y se publicó originalmente en 1978, fue el primer estudio epidemiológico que examinó la relación entre la dieta y las ECV en diferentes poblaciones y partes del mundo. Sus resultados influyeron en las recomendaciones nutricionales en ECV a nivel mundial y en la consolidación de la creencia de que una mayor ingesta de colesterol, grasas totales y ácidos grasos saturados se asociaba directamente con una mayor prevalencia de ECV.
Las Guías Alimentarias Estadounidenses (1980), elaboradas por el Departamento de Agricultura de Estados Unidos y por el Departamento de Salud y Servicios Humanos, se mantuvieron centradas en nutrientes e indicaban principalmente evitar el exceso de grasas, grasas saturadas y colesterol; consumir alimentos con almidón y fibra adecuada; evitar el exceso de azúcar y el exceso de sodio (9). Estas directrices dietéticas impulsaron las políticas de nutrición a nivel mundial y en la mayoría de las naciones occidentales, y algunos países de Latinoamérica adoptaron posteriormente consejos similares. Al igual que la pirámide de alimentos del Departamento de Agricultura de Estados Unidos, las pautas derivadas de las guías alimentarias de Estados Unidos han sido criticadas por estar excesivamente influenciadas por la industria agrícola, alimentaria y farmacéutica (10).

Así, el tratamiento nutricional y la prevención de las ECV en sus inicios se centraron en la reducción de las calorías, grasas y colesterol y, paralelamente, en la creación de nuevos productos alimenticios para dar respuesta a esta demanda, tales como alimentos procesados bajos en grasas y calorías, la irrupción de los edulcorantes y de carbohidratos de alta carga glucémica (11).

Numerosos ensayos aleatorizados y estudios epidemiológicos revisaron las pautas dietéticas mencionadas previamente y cuestionaron sus conclusiones. Entre ellos se destacan un metaanálisis (12) y dos revisiones, que no confirmaron en sus hallazgos la asociación entre las grasas saturadas y las ECV $(13,14)$. Además, varios estudios, entre ellos el estudio epidemiológico prospectivo urbano y rural "The Prospective Urban Rural Epidemiology study (PURE)" (15), un estudio a gran escala, evidenciaron que una dieta baja en grasas totales y grasas saturadas era ineficaz para combatir las ECV, obesidad, diabetes o cáncer. 
Con el correr de los años, los avances metodológicos en la ciencia de la nutrición pusieron de manifiesto que los enfoques en calorías y nutrientes no son suficientes y que las ECV —-fenómeno multicausal y complejo- están influenciadas por los efectos sinérgicos de alimentos y nutrientes, la calidad de los alimentos consumidos, su grado de procesamiento industrial y los patrones de la dieta en general $(2,16)$.

\section{Patrones alimentarios}

En los últimos años, se ha propuesto el análisis de los patrones alimentarios en el estudio de la relación alimentación-enfermedades crónicas, lo que permite tener una visión más general de la dieta que incluye la combinación global de alimentos y el efecto sinérgico de ciertos nutrientes $(17,18)$.

Conceptualmente, los patrones alimentarios representan "el consumo de diferentes alimentos, bebidas y nutrientes de la dieta, contemplando su variedad y combinación, como así también, la cantidad y frecuencia con que se consumen de manera habitual" (19, p. 9). Sin embargo, los patrones alimentarios pueden definirse de muchas maneras, entre ellas, "como una exposición o un comportamiento, como una construcción univariada o multivariada, basados en investigación 0 datos estáticos o dinámicos" (20, pp. 1,2). En general, los métodos comúnmente aplicados por los investigadores para examinar los patrones alimentarios pueden ser a priori, tales como los índices o scores (21), o a posteriori, incluyendo técnicas estadísticas multivariadas de reducción de datos, tales como análisis factorial, análisis de clúster y análisis de componentes principales (22). Los índices o scores miden la adherencia de la alimentación de una población a un patrón predefinido, por ejemplo, el nivel de adherencia a la dieta mediterránea "Mediterranean Diet Adherence Screener (MEDAS)" (23) o el score de adherencia a la dieta para detener la hipertensión "Dietary Approaches to Stop Hypertension (DASH)-style diet adherence score" (24); o la adherencia a recomendaciones alimentarias, por ejemplo, al índice de alimentación saludable Healthy Eating Index (HEI) (25), con base en una escala de puntuación basada en la frecuencia de consumo de los alimentos, tamaño y porciones, presencia de alimentos y nutrientes protectores, entre otros. Aunque la mayoría de estos índices se basan en alimentos 0 nutrientes, actualmente se han propuesto nuevos instrumentos para valorar la adherencia a estilos de vida saludable, incluyendo no solo el componente alimentario, sino también la actividad física, la interacción social y el estrés (26).

La literatura científica indica que la adherencia a determinados patrones alimentarios nutricionalmente equilibrados se asocia con un menor riesgo de ECV $(17,18)$. El estudio de los siete países fue el primer estudio epidemiológico que examinó la relación entre la dieta y las ECV en diferentes poblaciones y partes del mundo (8), como ya se mencionó, y puso en evidencia que los países con dietas ricas en alimentos de origen animal y azúcares pertenecientes al norte de Europa y de América tuvieron mayores tasas de mortalidad por enfermedad coronaria, mientras que los países con un consumo mayor de vegetales y pescados (costa mediterránea y Japón) presentaron menores tasas de mortalidad por cardiopatía coronaria (27). Con base a estos resultados, Ancel Keys publicó en su libro How to Eat well and Stay well the mediterranean way (Como comer bien $y$ mantenerse sano al estilo mediterráneo) las propiedades y los beneficios de esta forma de vida, acuñando el concepto de dieta mediterránea (28).

El patrón alimentario mediterráneo junto al DASH son los patrones más estudiados en relación con las enfermedades crónicas y, particularmente, con las ECV (2). La dieta mediterránea comprende 
una alta ingesta de frutas, verduras, legumbres, frutos secos, semillas, cereales integrales y aceite de oliva; una ingesta moderada de pescado, aves de corral y huevo; bajo consumo de carnes rojas y procesadas, consumo ocasional de alimentos dulces y postres; y una ingesta moderada de vino tinto. En relación con los ensayos clínicos, el estudio de la dieta del corazón de Lyon, "The Lyon Diet Heart Study", fue el primer trabajo sobre prevención secundaria de eventos coronarios. En este estudio, De Lorgeril et at. (29) hallaron que el patrón mediterráneo se asoció con una menor tasa de recurrencia después de un primer infarto de miocardio. El estudio prevención con dieta mediterránea, "Prevention with Mediterranean Diet" (PREDIMED), un ensayo multicéntrico realizado en España, puso de manifiesto que la dieta mediterránea suplementada con aceite extra virgen de oliva o frutos secos reduce la incidencia de eventos cardiovasculares en personas con factores de riesgo (30). Resultados preliminares del estudio de intervención dietaria coronaria con aceite de oliva y prevención cardiovascular, "Coronary Diet Intervention with Olive oil and Cardiovascular Prevention" (CORDIOPREV), un ensayo clínico reciente sobre prevención secundaria de ECV, mostró que la adherencia a una dieta mediterránea rica en aceite de oliva extravirgen mejora la función endotelial de pacientes diabéticos y prediabéticos (31). De igual manera, los estudios observacionales realizados a gran escala han reportado el efecto cardioprotector del patrón mediterráneo (32).

El patrón alimentario DASH, basado fundamentalmente en la ingesta de cereales integrales, verduras, frutas, legumbres, nueces, carnes magras, lácteos con bajo contenido en grasa y un aporte de sodio limitado, ha demostrado ser eficaz en la disminución de la presión arterial (33). Aunque la dieta DASH tiene muchas similitudes con la mediterránea, Korakas et al. (4) sostienen que ambos patrones alimentarios se diferencian por el contenido en grasas, aportadas por el aceite de oliva en el patrón mediterráneo y por los productos lácteos en el DASH, además del contenido de sodio. Asimismo, diversos trabajos han demostrado que la adherencia a una dieta DASH se relaciona con otros beneficios para el sistema cardiovascular, tales como el descenso del peso corporal, de marcadores de inflamación y cardiometabólicos y una mejoría de la función endotelial y en la calidad de vida (34).

Otros patrones alimentarios, el occidental y el prudente, también han sido relacionados con las ECV. En el estudio prospectivo de salud de las enfermeras "Nurses' Health Study" (NHS), el patrón occidental, caracterizado por un consumo elevado de carnes rojas y procesadas, granos refinados y bebidas azucaradas, como también el consumo frecuente de alimentos AUP y comidas rápidas, se asoció con un mayor riesgo de enfermedad cardíaca coronaria, de accidente cerebrovascular y de mortalidad por ECV, mientras que el patrón alimentario prudente (ingesta elevada de verduras, frutas, legumbres, pescado, aves y granos enteros) produjo un efecto opuesto (35). En el estudio de seguimiento de los profesionales de la salud, "Health Professionals Follow-up Study" (HPFS), se observó que la dieta occidental se asoció con un mayor riesgo de cardiopatía coronaria, por oposición al patrón alimentario prudente, que se correlacionó inversamente con el riesgo cardiovascular (36).

Es claro que los patrones alimentarios ricos en frutas, vegetales, fibra dietética y ácidos grasos mono y poliinsaturados podrían favorecer la disminución de la carga de ECV a través de diversos mecanismos, entre ellos, la disminución de biomarcadores lipídicos y de inflamación (33), la interacción con la expresión de genes involucrados en el metabolismo de la obesidad y un 
efecto positivo en la composición de la microbiota intestinal, en tanto que el consumo desmedido de alimentos ricos en grasas saturadas y trans, sodio y azúcares tendría un impacto negativo (37).

El análisis de los patrones alimentarios de una población constituye tanto un enfoque complementario y eficaz, en la epidemiología nutricional, apoyando la evidencia cardioprotectora de ciertos hábitos alimentarios, como también una base sólida para la formulación de recomendaciones y pautas alimentarias acordes con las necesidades individuales, preferencias socioculturales y estilos de vida locales. Si bien aún persiste el estudio del impacto de la dieta sobre las ECV basado en el análisis aislado de un solo alimento o nutrientes específicos, actualmente, la ciencia de los patrones alimentarios ha tomado auge, en aras de mejorar los aspectos metodológicos y conceptuales que permitan disminuir las brechas de investigaciones futuras, con mejor interpretación del efecto de la dieta en su totalidad, incluyendo la multidimensionalidad, el dinamismo, la combinación de comidas y preparaciones y el efecto sinérgico de los nutrientes sobre la promoción de la salud y la prevención de las enfermedades crónicas (38).

\section{Clasificación de alimentos según grado de procesamiento por sistema NOVA}

El sistema de clasificación NOVA, basado en el grado de procesamiento de alimentos, fue descrito de manera formal hace aproximadamente una década por Monteiro et al. (39) en la Universidad de San Pablo, Brasil. Este sistema agrupa los alimentos según el alcance y el propósito del procesamiento industrial (alimentos mínimamente procesados, ingredientes culinarios procesados, alimentos procesados y ultraprocesados) (3).
Los estudios basados en el sistema NOVA muestran que los AUP en la actualidad dominan el suministro de alimentos de varios países de altos ingresos y son cada vez más generalizados en los países de ingresos medios-bajos y medios-altos (40). La evidencia muestra que el desplazamiento de alimentos mínimamente procesados por AUP está asociado con perfiles de nutrientes dietéticos poco saludables y enfermedades crónicas relacionadas con la dieta (41). La OMS y el Fondo Mundial para la Investigación del Cáncer señalan que la proporción de AUP en los suministros de alimentos puede considerarse una medida de la calidad general de la alimentación de una población e influir en el desarrollo de obesidad, diabetes, ECV y varios tipos de cánceres $(40,41)$.

Según lo define el sistema de clasificación de alimentos NOVA, Ios AUP no son alimentos modificados o meramente procesados, sino que se trata de formulaciones industriales fabricadas a partir de sustancias derivadas de los alimentos, que suelen contener productos cosméticos, otros tipos de aditivos y escasos alimentos intactos (3). Las sustancias presentes en los AUP, como el jarabe de maíz alto en fructosa, carbohidratos de alta carga glucémica, edulcorantes no calóricos, potenciadores de sabor, emulsionantes, sodio, ácidos grasos trans y aceites vegetales hidrogenados, se encuentran asociadas a ECV por diferentes mecanismos, tales como resistencia a la insulina, inflamación, obesidad y, según hallazgos más recientes, por la modificación de la microbiota intestinal (42). Además, el procesamiento de alimentos a menudo implica la adición de una variedad de aceites vegetales que contienen un excedente relativo de ácidos grasos $\omega-6$, sobre el balance de ácidos grasos $\omega-3$. Este desbalance $\omega-6: \omega-3$ puede inducir a inflamación crónica de bajo grado a partir de la formación de eicosanoides con potencial inflamatorio, lo que contribuye en la progresión de la ECV (43). Por otra parte, los ácidos grasos trans también son un 
componente que se encuentra de manera frecuente en los AUP y se ha reportado a través de diversos estudios que existe un mayor riesgo de ECV al consumir niveles elevados de estos ácidos grasos, ya que están implicados en diversas vías inflamatorias, por lo que aumentan las concentraciones séricas de marcadores de inflamación, tales como la proteína $\mathrm{C}$ reactiva, la interleucina 6 y el factor de necrosis tumoral alfa, con impacto negativo en la función endotelial $(3,4)$.

La clasificación de los alimentos según su grado de procesamiento se considera en la actualidad de interés en las investigaciones relacionadas con la salud de la población y algunos investigadores sugieren su incorporación en la clasificación de alimentos para evaluar y monitorear los patrones alimentarios $(11,16)$.

Si bien la incorporación del análisis por sistema NOVA es de suma importancia, es la combinación global de alimentos que se consumen habitualmente o los patrones alimentarios los que en conjunto producen efectos sinérgicos de salud. Este enfoque, junto con el estudio de los entor- nos 0 ambientes alimentarios, es considerado el más completo para el análisis de los procesos de salud en alimentación (44). La siguiente figura esquematiza la historicidad en el enfoque y cambios de paradigma en el estudio de la alimentación y la ECV (Figura 1).

\section{CONCLUSIONES}

Si bien el análisis de la alimentación por nutrientes individuales ha funcionado para el tratamiento de las enfermedades de deficiencia, esto ha sido problemático para abordar las enfermedades crónicas, entre ellas las ECV. Es evidente que los factores dietéticos que afectan las ECV difieren de aquellos que conducen a las enfermedades carenciales, y su implicancia en estas últimas está relacionada con una ingesta 0 absorción nutricional disminuida, en tanto que el desarrollo de las ECV representa un proceso más complejo, de mayor duración, con la participación de múltiples componentes dietéticos integrados en un contexto de riesgo; por ejemplo, la exposición al humo del cigarrillo, el sedentarismo y el estrés crónico, entre otros.

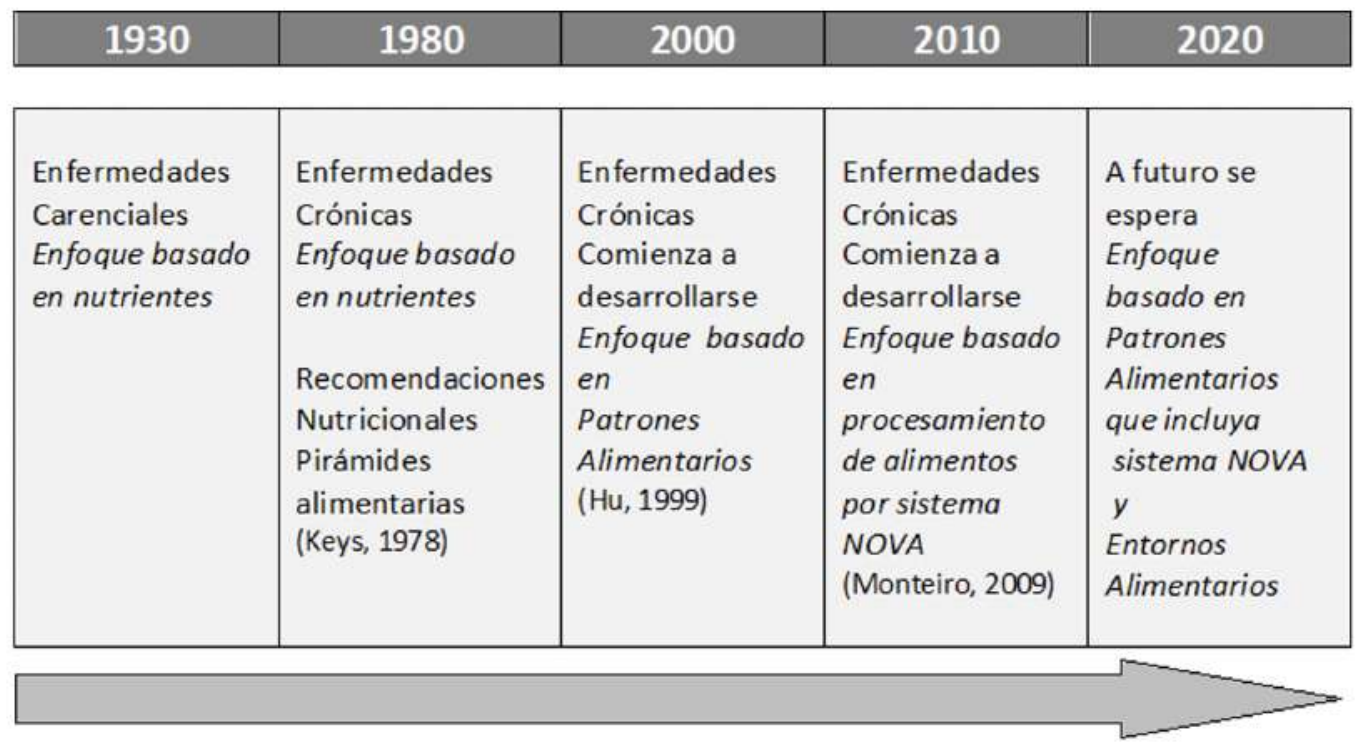

Figura 1. Transición histórica y cambios de paradigmas en el estudio de la alimentación y enfermedad cardiovascular. 
Aunque el enfoque reduccionista ha sido aplicado de manera histórica en los estudios nutricionales, este presenta ciertas limitaciones, pues supone que un componente dietético o alimento actúa de manera aislada en la fisiopatología de la enfermedad y asume el efecto absoluto del nutriente en estudios sobre el riesgo cardiovascular. Con base en esto, durante mucho tiempo se han postulado diferentes intervenciones alimentarias tendientes a mejorar la salud cardiovascular centradas en los macro 0 micronutrientes, sin contemplar el patrón alimentario 0 , más bien, el patrón de estilo de vida de las personas.

En las últimas décadas, la epidemiología nutricional ha virado a favor del abordaje nutricional de las ECV desde un análisis multidimensional, representada por diferentes enfoques, entre ellos, los patrones alimentarios, el sistema de clasificación NOVA y entornos alimentarios. Si bien estos enfoques permiten un análisis integrador u holístico, con una visión más amplia de la realidad alimentaria-nutricional, y contemplan la totalidad de la dieta y la interrelación biológica entre los nutrientes presentes en los alimentos, aún se requiere una mejor definición en lo que respecta a los aspectos metodológicos. En este sentido, los instrumentos de evaluación de la dieta, los métodos de construcción de los patrones alimentarios y el análisis estadístico pueden variar entre investigadores y, en algunos casos, ser complejos. Es por ello que se enfatiza en la estandarización de los diseños de estudios para mejorar la comprensión del impacto de ciertos patrones sobre la salud y las enfermedades crónicas.

Sin duda, el cambio de paradigma en el abordaje nutricional de las ECV se traduce en cambios positivos, entre ellos, una mejora en el análisis de las investigaciones en nutrición y el relevamiento de datos, el diseño de tablas de composición química con base en la evidencia local, la formación de recursos humanos en salud con visión crítica desde un para- digma integral, la promoción de recomendaciones alimentarias y la elaboración de guías nacionales que contemplen la alimentación tradicional y las costumbres locales, la reglamentación de la venta y publicidad de alimentos, el cuidado del medioambiente con base en la alimentación consciente y la promoción de patrones de alimentación saludables y adecuados a cada entorno, considerando el procesamiento industrial de los alimentos y su impacto a nivel individual y global.

En el proceso de reflexión no se realizó un metaanálisis de los resultados reportados por la alta heterogeneidad de los estudios mencionados, lo cual constituye una limitación de este trabajo. Sin embargo, se propuso un abordaje conceptual novedoso acerca de la historicidad en el abordaje clínico-nutricional de las ECV, como un primer paso para futuras investigaciones en el área.

La reflexión desarrollada pretende manifestar que, aunque aún existen diversos desafíos en el estudio de las ECV, a futuro se espera el desarrollo de instrumentos de evaluación de los entornos alimentarios con la inclusión de factores emergentes tales como contaminantes ambientales, disruptores endocrinos, cambios en la composición corporal, conductas sedentarias y tiempo en pantalla, publicidad y marketing de alimentos, ritmos circadianos, descanso y estrés crónico, a fin de avanzar en el campo de la epidemiología nutricional y ampliar el análisis del fenómeno multidimensional de las ECV, con resultados centrados en la promoción de estilos de vida y no en "grasas" o "carbohidratos".

\section{CONFLICTO DE INTERESES}

Se declara que el manuscrito no contiene material protegido por derecho de reproducción ni genera conflictos de interés. 


\section{---Referencias}

1. OMS. Enfermedades cardiovasculares. 2017. [Internet]. [Citado julio de 2019]. Disponible en:https://www.who.int/es/newsroom/fact-sheets/detail/cardiovascular-diseases-(cvds)

2. Mozaffarian D. Dietary and policy priorities for cardiovascular disease, diabetes, and obesity: A comprehensive review. Circulation. 2016;133:187-225. https://doi.org/10.1161/CIRCULATIONAHA.115.018585

3. Monteiro CA, Cannon G, Levy RB, Moubarac JC, Louzada ML, Rauber F, et al. Ultra-processed foods: What they are and how to identify them. Public Health Nutr. 2019;22:936-41. https://doi.org/10.1017/S1368980018003762

4. Korakas E, Dimitriadis G, Raptis A, Lambadiari V. Dietary composition and cardiovascular risk: A mediator or a bystander? Nutrients. 2018;10:1912. https://doi.org/10.3390/nu10121912

5. Davis C, Saltos E. Dietary recommendations and how they have changed over time. America's Eating Habits: Changes and Consequences. Washington, DC: US Department of Agriculture, Economic Research Service; 1999. Agriculture Information Bulletin N. ${ }^{\circ} 750$.

6. Konstantinov IE, Jankovic GM, Alexander I. Ignatowski: A pioneer in the study of atherosclerosis. Tex Heart Inst J. 2013;40:246-49. Disponible en: https://pubmed.ncbi.nlm.nih.gov/23914012/

7. Dawber TR, Meadors GF, Moore FE Jr. Epidemiological approaches to heart disease: the Framingham Study. Am J Public Health Nations Health. 1951;41(3):279-81. https://doi.org/10.2105/ajph.41.3.279

8. Keys A, Menotti A, Karvonen MJ, Aravanis C, Blackburn H, Buzina R, et al. The diet and 15-year death rate in the seven countries study. Am J Epidemiol. 1986;124:90315. https://doi.org/10.1093/oxfordjournals.aje.a114480

9. Dietary Guidelines Advisory Committee. Scientific Report of the 2015 Dietary Guidelines Advisory Committee. 2015. [Internet]. [Citado agosto de 2019]. Disponible en: http://www.health.gov/dietaryguidelines/2015-scientific-report/

10. Teicholz N. The scientific report guiding the US dietary guidelines: Is it scientific? BMJ. 2015;351:h4962. https://doi.org/10.1136/bmj.h4962

11. Fardet A. characterization of the degree of food processing in relation with its health potential and effects. Adv Food Nutr Res. 2018;85:79-129. https://doi.org/10.1016/bs.afnr.2018.02.002

12. Siri-Tarino PW, Sun Q, Hu FB, Krauss RM. Saturated fat, carbohydrate, and cardiovascular disease. Am J Clin Nutr. 2010;91:502-9. https://doi.org/10.3945/ajcn.2008.26285

13. Skeaff $\mathrm{CM}$, Miller J. Dietary fat and coronary heart disease: summary of evidence from prospective cohort and randomised controlled trials. Ann Nutr Metab. 2009;55:173-201. https://doi.org/10.1159/000229002

14. Hooper L, Summerbell CD, Thompson R, Sills D, Roberts FG, Moore HJ, et al. Reduced or modified dietary fat for preventing cardiovascular disease. Cochrane Database Syst Rev.2011;6;(7):CD002137. https://doi.org/10.1002/14651858.CD002137.pub3

15. Dehghan M, Mente A, Zhang X, Swaminathan S, Li W, Mohan V, et al. Associations of fats and carbohydrate intake with cardiovascular disease and mortality in 18 countries from five continents (PURE): A prospective cohort study. Lancet. 2017;390:2050-62. https://doi.org/10.1016/S0140-6736(17)32252-3

16. Monteiro CA, Cannon G, Moubarac JC, Levy RB, Louzada ML, Jaime PC. The UN Decade of Nutrition, the NOVA food classification and the trouble with ultra-processing. Public Health Nutr. 2018;21:5-17. https://doi.org/10.1017/S1368980017000234

17. Marchiori GN, González AL, Perovic NR, Defagó MD. Una mirada global sobre la influencia de los patrones alimentarios en las enfermedades cardiovasculares. Perspect Nutr Humana. 2017;19:79-92. https://doi.org/10.17533/udea.penh.v19n1a07 


\section{Nutrientes, patrones alimentarios y enfermedad cardiovascular}

18. Defagó MD, Elorriaga N, Irazola VE, Rubinstein AL. Influence of food patterns on endothelial biomarkers: a systematic review. J Clin Hypertens. 2014;16:907-13. https://doi.org/10.1111/jch.12431

19. United States Department of Agriculture. A series of systematic reviews on the relationship between dietary patterns and health outcomes. 2014. [Internet]. [Citado agosto de 2019]. Disponible en: https://www.cnpp.usda.gov/nutrition-evidencelibrary-dietary-patterns-systematic-review-project

20. Reedy J, Subar AF, George SM, Krebs-Smith SM. Extending methods in dietary patterns research. Nutrients. 2018;10:571. https://doi.org/10.3390/nu10050571

21. Waijers PM, Feskens EJ, Ocké MC. A critical review of predefined diet quality scores. Br J Nutr.2007;97:219-31. https://doi.org/10.1017/S0007114507250421

22. Gleason PM, Boushey CJ, Harris JE, Zoellner J. Publishing nutrition research: A review of multivariate techniques-part 3: Data reduction methods. J Acad Nutr Diet.2015;115:1072-82. https://doi.org/10.1016/j.jand.2015.03.011

23. Schröder H, Fitó M, Estruch R, Martínez-González MA, Corella D, Salas-Salvadó J, et al. A short screener is valid for assessing Mediterranean diet adherence among older Spanish men and women. J Nutr. 2011;141:1140-5. https://doi.org/10.3945/ jn.110.135566

24. Fung TT, Chiuve SE, McCullough ML, Rexrode KM, Logroscino G, Hu FB. Adherence to a DASH-style diet and risk of coronary heart disease and stroke in women. Arch Intern Med. 2008;168:713-20. https://doi.org/10.1001/archinte.168.7.713

25. Sanjeevi N, Lipsky LM, Nansel TR. Cardiovascular biomarkers in association with dietary intake in a longitudinal study of youth with type 1 diabetes. Nutrients. 2018;10:1552. https://doi.org/10.3390/nu10101552

26. Sotos-Prieto M, Moreno-Franco B, Ordovás JM, León M, Casasnovas JA, Peñalvo JL. Design and development of an instrument to measure overall lifestyle habits for epidemiological research: The Mediterranean Lifestyle (MEDLIFE) index. Public Health Nutr. 2015;18:959-67. https://doi.org/10.1017/S1368980014001360

27. Menotti A, Kromhout D, Blackburn H, Fidanza F, Buzina R, Nissinen A. Food consumption patterns and 25-year mortality from coronary heart disease: Cross-cultural correlations in the Seven Countries Study. The Seven Countries Study Research Group. Eur J Epidemiol. 1999;15:507-15. https://doi.org/10.1023/A:1007529206050

28. Keys A, Keys M. How to eat well and stay well the mediterranean way. New York: Doubleday, 1975.

29. De Lorgeril M, Salen P, Martin JL, Monjaud I, Delaye J, Mamelle N. Mediterranean diet, traditional risk factors, and the rate of cardiovascular complications after myocardial infarction: Final report of the Lyon Diet Heart Study. Circulation. 1999;99:779-85. https://doi.org/10.1161/01.CIR.99.6.779

30. Estruch R, Ros E, Salas-Salvadó J, Covas MI, Corella D, Arós F, et al. Primary prevention of cardiovascular disease with a Mediterranean diet. N Engl J Med.2013;368:1279-90. https://doi.org/10.1056/NEJMoa1200303

31. Torres-Peña JD, Garcia-Rios A, Delgado-Casado N, Gomez-Luna P, Alcala-Diaz JF, Yubero-Serrano EM, et al. Mediterranean diet improves endothelial function in patients with diabetes and prediabetes: A report from the CORDIOPREV study. Atherosclerosis.2018;269:50-6. https://doi.org/10.1016/j.atherosclerosis.2017.12.012

32. Panagiotakos DB, Georgousopoulou EN, Pitsavos C, Chrysohoou C, Skoumas I, Pitaraki E, et al. Exploring the path of Mediterranean diet on 10-year incidence of cardiovascular disease: the ATTICA study (2002-2012). Nutr Metab Cardiovasc Dis.2015;25:327-35. https://doi.org/10.1016/j.numecd.2014.09.006

33. Appel LJ, Moore TJ, Obarzanek E, Vollmer WM, Svetkey LP, Sacks FM,et al. A clinical trial of the effects of dietary patterns on blood pressure. DASH Collaborative Research Group. N Engl J Med. 1997;336:1117-24. https://doi.org/10.1056/NEJM199704173361601 
34. Phillips CM, Harrington JM, Perry IJ. Relationship between dietary quality, determined by DASH score, and cardiometabolic health biomarkers: A cross-sectional analysis in adults. Clin Nutr.2019;38:1620-8. https://doi.org/10.1016/..clnu.2018.08.028

35. Fung TT, Willett WC, Stampfer MJ, Manson JE, Hu FB. Dietary patterns and the risk of coronary heart disease in women. Arch Intern Med. 2001;161:1857-62. https://doi.org/10.1001/archinte.161.15.1857

36. Hu FB, Rimm EB, Stampfer MJ, Ascherio A, Spiegelman D, Willett WC. Prospective study of major dietary patterns and risk of coronary heart disease in men. Am J Clin Nutr. 2000;72:912-21. https://doi.org/10.1093/ajcn/72.4.912

37. Morera LP, Marchiori GN, Medrano LA, Defagó MD. Stress, dietary patterns and cardiovascular disease: A mini-review. Front Neurosci. 2019;13:1226. https://doi:10.3389/fnins.2019.01226

38. Tapsell LC, Neale EP, Satija A, Hu FB. Foods, nutrients, and dietary patterns: Interconnections and implications for dietary guidelines. Adv Nutr. 2016;7:445-54. https://doi.org/10.3945/an.115.011718

39. Monteiro CA. Nutrition and health. The issue is not food, nor nutrients, so much as processing. Public Health Nutr. 2009;12:729-31. https://doi.org/10.1017/S1368980009005291

40. Organización panamericana de la salud y organización mundial de la salud (OPS/OMS). Alimentos y bebidas ultraprocesados en América Latina: tendencias, efecto sobre la obesidad e implicaciones para las políticas públicas. 2015. [Internet]. [Citado septiembre de 2019]. Disponible en: http://iris.paho.org/xmlui/bitstream/handle/123456789/7698/9789275318645_esp.pdf

41. Monteiro CA, Moubarac JC, Levy RB, Canella DS, Louzada M, Cannon G. Household availability of ultra-processed foods and obesity in nineteen European countries. Public Health Nutr. 2018;21:18-26. https://doi.org/10.1017/S1368980017001379

42. Swithers S. Artificial sweeteners produce the counterintuitive effect of inducing metabolic derangements. Trends Endocrinol Metab. 2013; 24:431-41. https://doi.org/10.1016/.tem.2013.05.005

43. Simopoulos AP. The importance of the omega-6/omega-3 fatty acid ratio in cardiovascular disease and other chronic diseases. Exp Biol Med (Maywood). 2008;233:674-88. https://doi.org/10.3181/0711-MR-311

44. Siri-Tarino PW , Krauss RM . Diet, lipids, and cardiovascular disease. Curr Opin Lipidol. 2016;27:323-8. https://doi.org/10.1097/ MOL.0000000000000310 